\title{
Traumatismo penetrante cardiaco: caracterización, resultados inmediatos y variables asociadas a morbilidad y mortalidad en pacientes operados
}

\author{
Roberto González L., ${ }^{1,2}$, Alejandra Riquelme U. ${ }^{2}$, Alberto Fuentes E. ${ }^{3}$, \\ Jorge Canales Z. ${ }^{3}$, Enrique Seguel S. ${ }^{1,2}$, Aleck Stockins L. ${ }^{1,2}$, Andrés Jadue T. ${ }^{1}$, \\ Rodrigo Reyes M. ${ }^{2}$, René Saldías F. ${ }^{4}$ y Emilio Alarcón C. ${ }^{1,2}$
}

Penetrating cardiac trauma: findings, outcomes and prognostic factors in operated patients

Introduction: Penetrating cardiac trauma (PCT) constitute a permanent surgical challenge due to it characteristics and high mortality. Aim: To describe the findings, outcomes and prognostic factors in PCT. Materials and Method: Cross-sectional descriptive study, review of prospective thoracic trauma protocols and surgical registries. Period January 1990-December 2017. All patients with PCT were included. Various variables are described and analyzed. Univariate and multivariate analysis were performed to identify factors associated with morbidity, neurologic dysfunction and mortality. Results: 220 patients PCT, 209 (95.0\%) men, mean age $30.4 \pm 13.3$, median 27 years. Mechanism: Aggression in $202(91.8 \%)$. The traumatic agent was cold steel in $186(84.4 \%)$ and fire arm in $21(9.5 \%) .169(76.8 \%)$ patients presented with tamponade, $103(46.8 \%)$ shock and $20(9.1 \%)$ cardiopulmonary arrest. Approach was sternotomy in $157(71.4 \%)$, left thoracotomy in $58(26.4 \%)$. The most common injured areas were right ventricle in $110(50.0 \%)$, left ventricle in $72(32.7 \%) .135(61.4 \%)$ patients needed postoperative ICU and $74(33.6 \%)$ were transfused. Complications occurred in 60 (27.3\%). Twenty-one (9.5\%) were reoperated. Mortality 28 (12.7\%). The postoperative median stay was 6 days (Range 1-150). Prognostic factors were identified. Discussion: Operated PCT are more frequent in men with stab wound, the most common injured area is the right ventricle. The morbidity and mortality is comparable with international series.

Key words: cardiac surgery; cardiac tamponade; thoracic trauma.

\section{Resumen}

Introducción: El traumatismo penetrante cardiaco (TPC) por sus características y en particular por su alta mortalidad, constituye un desafío quirúrgico permanente. Objetivos: Describir las características, resultados inmediatos y factores pronósticos en TPC. Materiales y Método: Estudio descriptivo transversal, revisión de protocolos prospectivos de traumatismo torácico, registros de pabellón y fichas clínicas. Período enero de 1990-diciembre de 2017. Se incluyeron todos los pacientes con TPC operados. Se describen y analizan diversas variables. Se realizó regresión logística con análisis univariado y multivariado para identificar variables asociadas a morbilidad, deterioro neurológico y mortalidad. Resultados: 220 pacientes operados por TPC, $209(95,0 \%)$ hombres, edad promedio 30,4 $\pm 13,3$, mediana 27 años. Mecanismo: agresión en 202 (91,8\%). El agente traumático fue en $186(84,5 \%)$ arma blanca, en $21(9,5 \%)$ arma de fuego. Presentaban taponamiento $169(76,8 \%)$ pacientes, ingresaron en shock $103(46,8 \%)$ y en paro cardiorrespiratorio $20(9,1 \%)$. Vía de abordaje fue esternotomía en $157(71,4 \%)$, toracotomía izquierda en $58(26,4 \%)$. Las cavidades cardiacas lesionadas más frecuentes fueron ventrículo derecho en $110(50,0 \%)$, ventrículo izquierdo en $72(32,7 \%)$. Se hospitalizaron en UCI en el postoperatorio $135(61,4 \%)$, se transfundieron $74(33,6 \%)$, presentaron complicaciones $60(27,3 \%)$ y se reoperaron $21(9,5 \%)$. Mortalidad 28 (12,7\%). La estadía postoperatoria tuvo una mediana de 6 días (rango 1-150). Se identificaron factores pronósticos. Discusión: Los TPC operados son más frecuentes en hombres agredidos con arma blanca, la cavidad lesionada más frecuente es el ventrículo derecho. Nuestra morbimortalidad es comparable con series internacionales.

Palabras clave: cirugía cardiaca; taponamiento cardiaco; traumatismo torácico. 


\section{Introducción}

Desde la primera descripción del traumatismo penetrante cardiaco (TPC) en los tiempos de la Ilíada, éste ha sido considerado una lesión con características especiales. El corazón durante muchos años fue considerado un órgano noble e inoperable, incluso el reconocido cirujano Theodor Billroth, en el Congreso de Cirugía de 1883, proclamó: "el cirujano que intente reparar una lesión cardiaca debe perder el respeto de sus colegas". No fue hasta septiembre de 1896, en Frankfurt, cuando el jardinero Wilhelm Justus, tras sufrir un TPC por arma blanca en el ventrículo derecho y ser tratado con medidas de mantención con una bolsa de hielo y alcanfor por el asistente del cirujano Ludwig Rehn (que se encontraba de viaje), fuera el primer paciente intervenido quirúrgicamente con éxito. Al regresar Rehn decide operarlo, logrando marcar el 9 de septiembre de 1896 como el inicio de la cirugía en TPC. Desde ahí, ha sido considerado un desafío quirúrgico permanente, por su alta morbimortalidad y tratamiento de urgencia ${ }^{1,2}$.

El TPC afecta principalmente a hombres jóvenes previamente sanos, secundario a una herida por arma blanca o arma de fuego, pero también por causas iatrogénicas como cateterización de cavidades, instalación de drenajes torácicos, entre otros ${ }^{3,4}$. De acuerdo al tipo de agente traumático y mecanismo se pueden producir diferentes lesiones cardiacas, desde desgarros de la pared libre y lesiones de grandes vasos intrapericárdicos hasta lesiones coronarias 0 intracavitarias afectando septum, válvulas o incluso alteraciones del sistema de conducción ${ }^{3}$.

La mayoría de la mortalidad asociada al TPC (60-80\%) ocurre en el sitio del traumatismo o antes de la llegada del paciente a los servicios de urgencia, aumentado la supervivencia considerablemente (27-85\%) al llegar a estos ${ }^{4,5}$. La supervivencia ha aumentado en los últimos años gracias a los avances en los cuidados prehospitalarios, rapidez de traslado $\mathrm{y}$ avances perioperatorios ${ }^{4}$.

Las manifestaciones clínicas del TPC no siempre se pueden consignar, ya que un grupo importante no sobrevive al traslado o ingresa inmediatamente para un tratamiento quirúrgico definitivo. Pese a esto, se ha descrito que el TPC puede ir desde un cuadro totalmente asintomático hasta shock o colapso cardiovascular, por lo que su diagnóstico se basa en la clínica más el antecedente de un traumatismo torácico generalmente precordial. El taponamiento cardiaco es la forma más frecuente de presentación $^{3,4}$. Se han descrito variables que se asocian a un peor pronóstico.
Nuestros objetivos son describir las características y resultados inmediatos de los pacientes operados por TPC en nuestro hospital e identificar las variables asociadas a morbilidad, deterioro neurológico permanente y mortalidad.

\section{Material y Método}

Se realizó estudio descriptivo transversal de los pacientes operados en el Hospital Clínico Regional "Dr. Guillermo Grant Benavente" de Concepción, Chile, en el período comprendido entre enero de 1990 y diciembre de 2017. Se incluyeron todas las cirugías por TPC. La información se obtuvo a partir de protocolos prospectivos de TPC realizados al ingreso, fichas clínicas, registros de pabellón y protocolos quirúrgicos.

Se utilizó planilla Microsoft Excel ${ }^{\circledR}$ y software SPSS 24 para la tabulación de datos y posterior análisis. Se describen sexo, edad, agente traumático, asociación con: taponamiento cardiaco, paro cardiorrespiratorio (PCR), otras lesiones intratorácicas y extratorácicas, abordaje, hallazgos intraoperatorios, ingreso a cuidados intensivos (UCI), morbilidad y mortalidad. Para búsqueda de variables asociadas a morbilidad, deterioro neurológico y mortalidad se realizó un análisis univariado con cálculo de Odds Ratio. Se consideró estadísticamente significativo $\mathrm{p}<0,05$. Para el análisis multivariado consideramos a aquellas con asociación significativa en el análisis univariado.

Se consideró politraumatizado aquel paciente con lesiones en 2 o más sistemas y con Injury Severity Score $\left(\right.$ ISS) $>16$ pts $^{6,7}$.

\section{Resultados}

En el período se hospitalizaron 3.640 traumatismos torácicos y se operaron $220(6,0 \%)$ TPC. Fueron $209(95,0 \%)$ hombres, con una relación hombre/ mujer: 19/1. La edad promedio fue de 30,4 $\pm 13,3$ años (Tabla 1).

El principal mecanismo del traumatismo fue agresión en $202(91,8 \%)$ pacientes. El agente traumático más frecuente fue el arma blanca en 186 $(84,5 \%)$ pacientes (Tabla 1$)$.

Desde el ingreso se consignó taponamiento cardiaco en 169 (76,8\%) pacientes, shock en 103 $(46,8 \%)$ y tríada de Beck en $76(34,5 \%)$ (Tabla 2$)$. Se estudió con ecocardiografía preoperatoria a 29 $(13,2 \%)$ pacientes y se realizaron $8(3,6 \%)$ ventanas subxifoideas. 
Tabla 1. Edad, sexo, mecanismo y agente del traumatismo penetrante cardiaco

\begin{tabular}{|c|c|}
\hline Total traumatismo penetrante cardiaco & 220 \\
\hline Edad promedio (años) & $30,4 \pm 13,3$ \\
\hline Mediana & 27 \\
\hline Rango & $11-87$ \\
\hline \multicolumn{2}{|l|}{ Sexo } \\
\hline Hombre & $209(95,0 \%)$ \\
\hline Mujer & $11(5,0 \%)$ \\
\hline \multicolumn{2}{|l|}{ Mecanismo } \\
\hline Agresión & $202(91,8 \%)$ \\
\hline Iatrogenia & $12(5,5 \%)$ \\
\hline Autoinferida & $5 \quad(2,3 \%)$ \\
\hline Caída sobre trozo de madera & $1(0,5 \%)$ \\
\hline \multicolumn{2}{|l|}{ Agente } \\
\hline Arma blanca & $186(84,5 \%)$ \\
\hline Arma de fuego & $21 \quad(9,5 \%)$ \\
\hline Catéter endocavitario & $9(4,1 \%)$ \\
\hline Tubo endopleural & $2(0,9 \%)$ \\
\hline Punción pericárdica & $1 \quad(0,5 \%)$ \\
\hline Trozo de madera & $1 \quad(0,5 \%)$ \\
\hline
\end{tabular}

Lesiones y/o hallazgos asociados se detectaron en $110(50,0 \%)$ pacientes, de los cuales fueron intratorácicos en $97(44,1 \%)$ y extratorácicos en 30 $(13,6 \%)$, siendo lo más frecuente hemotórax en 75 $(34,1 \%)$ y lesión abdominal en $18(8,2 \%)$ respectivamente. Se asociaron a lesiones tanto intra como extratorácicas $16(7,3 \%)$ pacientes (Tabla 2$)$.

El abordaje utilizado fue esternotomía en 157 $(71,4 \%)$ y toracotomía en $63(28,6 \%)$, entre estas, 57 toracotomías izquierdas y 6 derechas (Tabla 3 ). Se realizaron 2 toracotomías de urgencias en box para luego ingresar a tratamiento quirúrgico definitivo. No se presentó mortalidad ni déficit neurológico inmediato evidente en los 2 pacientes intervenidos por toracotomía de urgencia en box.

La cavidad cardiaca afectada más frecuentemente fue el ventrículo derecho en $110(50,0 \%)$ pacientes, presentándose concomitancia de 2 o más lesiones cardiacas en 28 (12,7\%) (Tabla 3$)$.

Se observó lesión intracardiaca en $11(5,0 \%)$, siendo $9(4,1 \%)$ shunt y $2(0,9 \%)$ lesión valvular, además, de $14(6,4 \%)$ con lesión coronaria asociada. En dos shunt no se realizó tratamiento quirúrgico, uno por rechazo del paciente y otro por comunicación interventricular pequeña sin significancia clínica. Una lesión valvular mínima, atribuida al traumatismo, se detectó en el ecocardiograma
Tabla 2. Características clínicas descritas desde el ingreso y lesiones y/o hallazgos intratorácicos y extratorácicos asociados en pacientes con traumatismo penetrante cardiaco

\begin{tabular}{|lr|}
\hline Total traumatismo penetrante cardiaco & $\mathbf{2 2 0}$ \\
Características clínicas & \\
Taponamiento cardiaco & $169(76,8 \%)$ \\
Tonos cardiacos apagados & $136(61,8 \%)$ \\
Ingurgitación yugular & $126(57,3 \%)$ \\
Shock & $103(46,8 \%)$ \\
Tríada de Beck & $76(34,5 \%)$ \\
Escala de Glasgow $\leq 8$ pts. & $64(29,1 \%)$ \\
Paro cardiorrespiratorio & $20(9,1 \%)$ \\
Pulso paradojal & $7(3,2 \%)$ \\
Frotes pericárdicos & $3(1,4 \%)$ \\
Lesiones y/o hallazgos intratorácicos & \\
Hemotórax & $75(34,1 \%)$ \\
Lesión parénquima pulmonar & $25(11,4 \%)$ \\
Neumotórax & $24(10,9 \%)$ \\
Lesión diafragmática & $4(1,8 \%)$ \\
Lesión TABC & $1(0,5 \%)$ \\
Lesión de tráquea & $1(0,5 \%)$ \\
Lesiones y/o hallazgos extratorácicos & \\
Abdominal & $18(8,2 \%)$ \\
Partes blandas & $4(1,8 \%)$ \\
Encefalocraneano & $3(1,4 \%)$ \\
Traumatismo óseo & $3(1,4 \%)$ \\
Traumatismo raquimedular & $2(0,9 \%)$ \\
Politraumatizado (ISS $>16$ pts.) & $18(8,2 \%)$ \\
\hline TAC: Tronco arteria broquioceálico; & \\
\hline
\end{tabular}

TABC: Tronco arterial braquiocefálico; ISS: Injury Severity Score.

Tabla 3. Abordaje quirúrgico y región anatómica lesionada por el traumatismo penetrante cardiaco

\begin{tabular}{|c|c|}
\hline Total traumatismo penetrante cardiaco & 220 \\
\hline Esternotomía & $157(71,4 \%)$ \\
\hline Toracotomía & $63(28,6 \%)$ \\
\hline $\begin{array}{l}\text { Izquierda } \\
\text { Derecha }\end{array}$ & $\begin{array}{r}57(25,9 \%) \\
6 \quad(2,7 \%)\end{array}$ \\
\hline \multicolumn{2}{|l|}{ Región anatómica } \\
\hline Ventrículo derecho & $110(50,0 \%)$ \\
\hline Ventrículo izquierdo & $72(32,7 \%)$ \\
\hline Aurícula derecha & $19(8,6 \%)$ \\
\hline Aurícula izquierda & $13(5,9 \%)$ \\
\hline Aorta & $11(5,0 \%)$ \\
\hline Cava inferior & $10(4,5 \%)$ \\
\hline Arteria pulmonar & $6(2,7 \%)$ \\
\hline Cava superior & $5(2,3 \%)$ \\
\hline
\end{tabular}

*Concomitancia de 2 o más lesiones cardiacas en 28 $(12,7 \%)$ pacientes. 


\section{ARTÍ́CULO ORIGINAL}

Tabla 4. Lesiones cardiacas asociadas al traumatismo penetrante cardiaco y su tratamiento quirúrgico

\begin{tabular}{|lcc|}
\hline Total traumatismo penetrante cardiaco & $\mathbf{2 2 0}$ & $\begin{array}{c}\text { Cirugía de lesión } \\
\text { cardiaca asociada }\end{array}$ \\
\hline Shunt & $9(4,1 \%)$ & $7(77,8 \%)$ \\
Comunicación interventricular & $6(2,7 \%)$ & $4(66,7 \%)$ \\
Aórtico-pulmonar & $2(0,9 \%)$ & $2(100 \%)$ \\
Aórtico-ventrículo derecho & $1(0,5 \%)$ & $1(100 \%)$ \\
Lesión valvular & $2(0,9 \%)$ & $1(50,0 \%)$ \\
Válvula tricúspide & $1(0,5 \%)$ & $1(100 \%)$ \\
Válvula mitral & $1(0,5 \%)$ & $0 \quad(0,0 \%)$ \\
Lesión arteria coronaria & $14(6,4 \%)$ & $4(28,6 \%)$ \\
Descendente anterior & $10(4,5 \%)$ & $4(40,0 \%)$ \\
Circunfleja & $3(1,4 \%)$ & $0 \quad(0,0 \%)$ \\
Derecha & $1(0,5 \%)$ & $0 \quad(0,0 \%)$ \\
\hline
\end{tabular}

*Todas las cirugías fueron realizadas por el equipo de Cirugía Cardiotorácica de nuestro hospital.
Tabla 5. Mortalidad, morbilidad, necesidad de reoperación y estadía postoperatoria asociada al traumatismo penetrante cardiaco

\begin{tabular}{|c|c|}
\hline Total traumatismo penetrante cardiaco & 220 \\
\hline Mortalidad & $28(12,7 \%)$ \\
\hline Intraoperatoria & $13(5,9 \%)$ \\
\hline Postoperatoria & $15(6,8 \%)$ \\
\hline Morbilidad & $60(27,3 \%)$ \\
\hline Hemotórax & $14(6,4 \%)$ \\
\hline Hemorragia & $13(5,9 \%)$ \\
\hline Déficit neurológico permanente & $12(5,5 \%)$ \\
\hline Neumonía & $7(3,2 \%)$ \\
\hline Infarto agudo al miocardio & $6(2,7 \%)$ \\
\hline Infección herida operatoria & $5(2,3 \%)$ \\
\hline Mediastinitis & $2(0,9 \%)$ \\
\hline Fístula bronco-pleural & $2(0,9 \%)$ \\
\hline Falla renal aguda & $2(0,9 \%)$ \\
\hline Otros & $6 \quad(2,7 \%)$ \\
\hline Reoperaciones & $21(9,5 \%)$ \\
\hline Hemorragia & $12(5,5 \%)$ \\
\hline Hemotórax coagulado & $7(3,2 \%)$ \\
\hline Mediastinitis & $2(0,9 \%)$ \\
\hline \multicolumn{2}{|l|}{ Estadía postoperatoria } \\
\hline Promedio (días) & $8,7 \pm 11,7$ \\
\hline Mediana & 6 \\
\hline Rango & $1-150$ \\
\hline \multicolumn{2}{|l|}{ Clavien-Dindo } \\
\hline I & 0 \\
\hline II & $19(8,6 \%)$ \\
\hline III & $30(13,6 \%)$ \\
\hline IV & $4 \quad(1,8 \%)$ \\
\hline $\mathrm{V}$ & $28(12,7 \%)$ \\
\hline
\end{tabular}

transfusiones (OR 4,91; IC 1,46-16,5; $\mathrm{p}=0,005$ ), tanto en el análisis univariado como el multivariado (Tablas 6 y 7).

Realizamos una regresión logística buscando variables asociadas a mortalidad. En un análisis univariado se asociaron significativamente el cursar con PCR (OR 31,0; IC 10,32-93,12; p =0,000), presencia de shock (OR 9,57, IC 3,19-28,68; $p=0,000)$, el sexo femenino (OR 4,41; IC 1,2-16,16; $p=0,016$ ), dos o más lesiones cardiacas (OR 3,87; IC 1,49$10,03 ; p=0,003)$, lesión extratorácica asociada (OR $2,72 ;$ IC $1,03-7,15 ; \mathrm{p}=0,037)$ y lesión de ventrículo izquierdo (OR 2,31; IC 1,04-5,15; p = 0,037). En el análisis multivariado se asociaron a mortalidad la presencia de shock, PCR, lesión extratorácica, más de una lesión y sexo femenino (Tablas 6 y 7). 
Tabla 6. Variables asociadas a morbilidad, deterioro neurológico permanente y mortalidad en análisis univariado

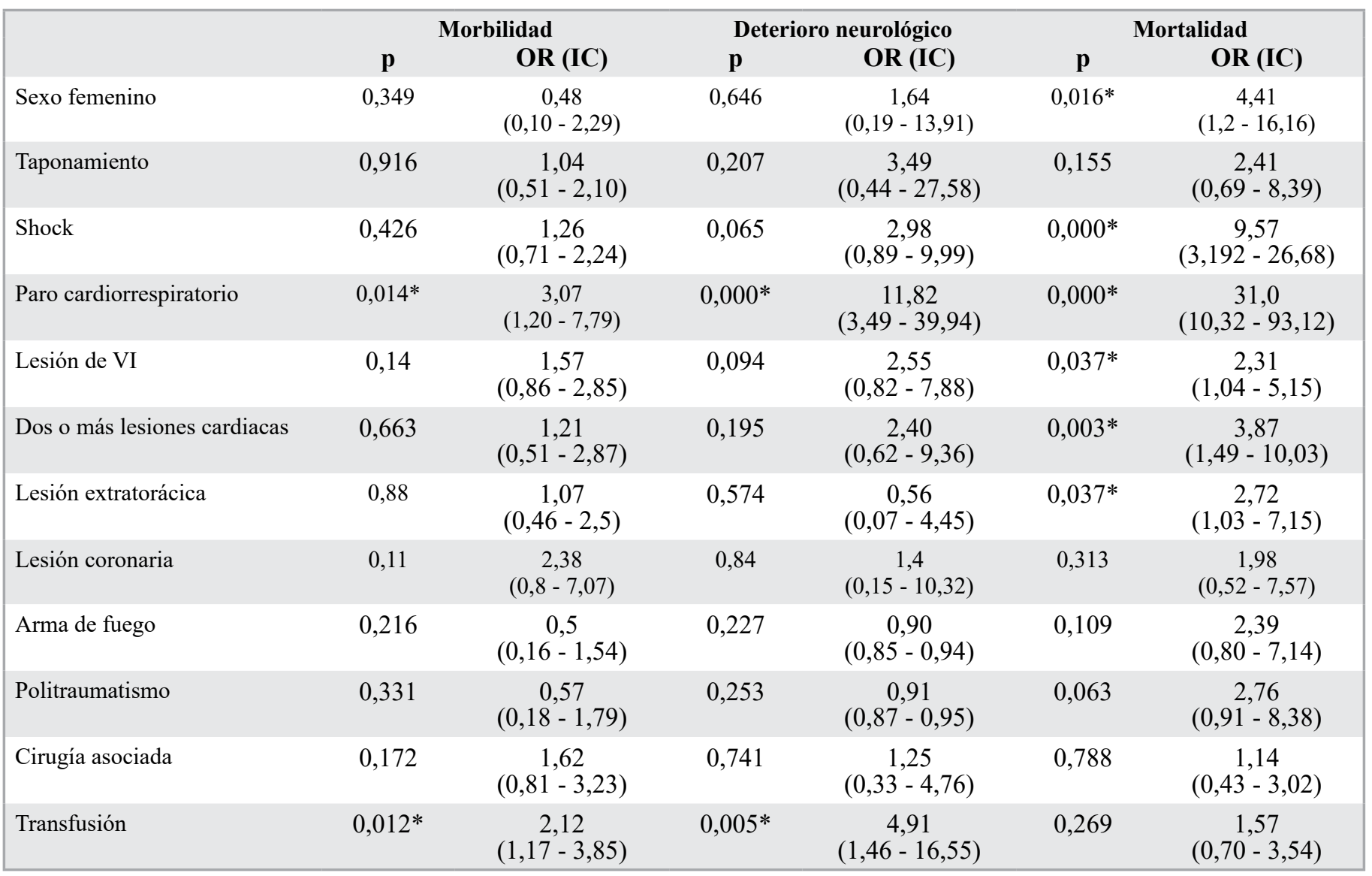

VI: Ventrículo izquierdo; OR: Odds Ratio; IC: Intervalo de confianza. *Estadísticamente significativo.

\section{Discusión}

El traumatismo cardiaco, tanto contuso como penetrante, es responsable de una significativa mortalidad, principalmente en pacientes jóvenes previamente sanos. A diferencia de lo que ocurre en nuestro medio, en donde el TPC es más frecuente, en Europa y en Estados Unidos, el traumatismo cardiaco contuso, principalmente secundario a un accidente de tránsito, es más común, ya sea por contusión miocárdica directa o por mecanismo de aceleración-deceleración ${ }^{3,4}$.

Se considera TPC cuando, secundario al traumatismo, ocurre una solución de continuidad de estructuras intrapericárdicas (cámaras cardiacas y grandes vasos intrapericárdicos), que presentan un comportamiento fisiopatológico, clínico, pronósticos y conductas terapéuticas similares, caracterizándose por demandar una rápida y oportuna intervención, debido a su alta morbimortalidad ${ }^{3,4}$.
Tabla 7. Variables asociadas a morbilidad, deterioro neurológico permanente y mortalidad en análisis multivariado

\begin{tabular}{|lcc|}
\hline & p & OR (IC) \\
Morbilidad & & \\
Paro cardiorrespiratorio & 0,001 & $5,191(1,962-13,735)$ \\
Transfusión & 0,047 & $2,065(1,009-4,226)$ \\
Deterioro neurológico & & \\
Paro cardiorrespiratorio & 0,000 & $12,584(3,451-45,883)$ \\
Transfusión & 0,012 & $5,244(1,440-19,104$ \\
Mortalidad & & \\
Sexo femenino & 0,021 & $7,143(1,337-38,157)$ \\
Shock & 0,004 & $6,184(1,906-21,167)$ \\
Paro cardiorrespiratorio & 0,000 & $46,21 \quad(11,48-185,93)$ \\
Dos o más lesiones cardiacas & 0,006 & $6,433(1,691-24,478)$ \\
Lesión extratorácica & 0,013 & $5,379(1,426-20,291)$ \\
\hline
\end{tabular}

OR: Odds Ratio; IC: Intervalo de confianza. 
En concordancia con diversas series, se presentó un predominio del sexo masculino, con edad promedio cercana a los 30 años. El mecanismo más frecuente es la agresión por arma blanca (28-94\%), sin embargo, en los últimos años ha aumentado la prevalencia de las agresiones por arma de fuego, provocando lesiones con mayor energía cinética, afectando a más de una cavidad cardiaca, además, de mayor riesgo de lesiones asociadas tanto intra como extratorácicas, relacionándose con una mayor mortalidad $^{4,5,8-11}$.

La presentación clínica más frecuente del TPC, que llega a la sala de urgencia, es el taponamiento cardiaco. El taponamiento cardiaco es un evento potencialmente mortal, a consecuencia de la acumulación de sangre u otro elemento en la cavidad pericárdica $^{12}$. Moreno el al., en una revisión de 100 pacientes con TPC, concluyó que la presencia de taponamiento cardiaco era un factor independiente de supervivencia, considerándolo como un "efecto protector" ${ }^{5,13,14}$. Algunas series describen la identificación de la tríada de Beck (tonos cardiacos apagados, ingurgitación yugular e hipotensión) para el diagnóstico del taponamiento cardiaco, sin embargo, es infrecuente de observar y consignar $(<15 \%)$, ya que la mayoría de los paciente con TPC ingresan a tratamiento quirúrgico sin un examen físico acucioso debido a su clara presentación y alta sospecha clínica ${ }^{5}$. En nuestra serie se consignó clínica de taponamiento cardiaco en el $76,8 \%$ y se consignó tríada de Beck en el 34,5\% de los pacientes.

En el traumatismo en general se han propuesto distintos scores con el objetivo de facilitar la comparación entre instituciones y la predicción de morbimortalidad. Dentro de ellos, el estado fisiológico o cardiovascular del paciente al momento del ingreso son predictores significativos del pronóstico del TPC $\mathrm{TP}^{4,5,9,15-18}$. En nuestra serie se observó un mayor número de pacientes en shock al ingreso que en publicaciones internacionales (32-34\%) y con un estado de conciencia (Escala de Glasgow $<8$ puntos) menor en comparaciones con otras series en que no supera el $10 \%{ }^{4,5}$. Es importante destacar, que a pesar de que solo 3 pacientes presentaron un traumatismo craneoencefálico asociado, este valor puede estar exagerado por la contribución del alcohol, drogas, traumatismo craneoencefálicos no identificados o sedación previa como ocurre en algunos pacientes con lesiones iatrogénicas.

Como se comentó anteriormente, el TPC, principalmente en presencia de taponamiento cardiaco o shock, corresponde una emergencia quirúrgica, que requiere un tratamiento oportuno ${ }^{4,5,10-13}$. En urgencia, el diagnóstico de taponamiento cardiaco se basa en el estado clínico, hemodinámico y el antecedente de traumatismo. Consideramos que la realización de la ventana pericárdica en pabellón quirúrgico, con todo preparado para la esternotomía, es una técnica bien estandarizada, simple, segura y con alta efectividad para el diagnóstico de un TPC en pacientes sin deterioro hemodinámico cuando se duda del diagnóstico. En nuestra serie se realizó ventana pericárdica en 8 $(3,6 \%)$ pacientes.

Si bien diversas series consideran la pericardiocentesis, que se define como una punción pericárdica con el objetivo de disminuir la presión intrapericárdica y evitar el colapso cardiovascular, como una opción terapéutica de rescate ${ }^{8,19-23}$, nuestro grupo no la reconoce ni considera una práctica útil como método diagnóstico ni terapéutico en TPC. En los últimos años diferentes publicaciones han demostrado un declive en el uso de este método. Morse y col, en su publicación mostró un descenso del uso de la pericardiocentesis desde un $35 \%$ en los años 1975-1985 hasta un 0\% en los años 20002010 y un descenso del uso de ventana pericárdica de un $34 \%$ al $10 \%$ en el mismo periodo ${ }^{4}$.

Uno de los avances más importantes en el diagnóstico en pacientes sin deterioro hemodinámico es la ecocardiografía, este permite observar hemopericardio, disfunciones miocárdicas, lesiones intracavitarias e incluso lesiones en la pared libre del corazón. Rozycki y col, demostraron una sensibilidad del $100 \%$ y una especificidad del $97,3 \%$ para el diagnóstico de derrames pericárdicos ${ }^{4,5,19-22}$. En nuestro medio el diagnóstico de TPC es fundamentalmente clínico, bastando el antecedente de traumatismo asociado a signos y síntomas de TPC, para plantear un tratamiento quirúrgico oportuno, realizándose ecocardiografía solo en pacientes sin deterioro hemodinámico y con duda diagnóstica, pero sin retrasar en ningún momento el tratamiento definitivo. Nosotros recomendamos realizar ecocardiografía postoperatoria para la detección de lesiones intracardiacas desapercibidas en el acto quirúrgico, que pueden estar presente hasta en un $5 \% 0^{4,5,24,25}$.

La presencia de lesiones asociadas tanto intratorácicas como extratorácicas se describe hasta en el $37-50 \%$ de los pacientes. Al comparar según el agente traumático se describe una mayor asociación de lesiones concomitantes en el traumatismo por arma de fuego que por arma blanca (42 vs 9\%), observándose principalmente lesiones en diafragma, pulmón e hígado. La mortalidad en pacientes con lesiones asociadas es significativamente superior que con lesiones aisladas del corazón ${ }^{4,5,8-11,21}$. En 
nuestra serie se presentó lesiones asociadas en 110 pacientes $(50 \%)$, principalmente hemotórax y lesiones abdominales.

Al igual que en distintas series, el ventrículo derecho corresponde a la cámara cardiaca más frecuentemente lesionada (37-60\%), seguido del ventrículo izquierdo (25-37\%), describiéndose lesión de arterias coronarias entre un $4-10 \%{ }^{4,5,8}$. Nuestro grupo realizó CRM asociada en el $28,6 \%$ de las lesiones coronarias, todas a la arteria descendente anterior (ADA) proximal. Al resto no se le realizó revascularización quirúrgica por ser lesiones pequeñas, distales y con escasa significancia clínica. Se describen lesiones de grandes vasos intrapericárdicos entre $3-30 \%{ }^{4,5,8}$, datos similares a nuestra serie con una sumatoria de $14,5 \%$ entre lesión de aorta, cavas y arteria pulmonar. Se presentó lesión intracavitaria en el 5\%, realizándose reparación de shunt y lesiones valvulares en $73 \%$ de los casos, todas realizadas por nuestro equipo.

El abordaje de elección es discutible, diferentes autores prefieren la toracotomía anterolateral, debido a su comodidad, facilidad y poca implementación permitiendo un acceso rápido $\mathrm{y}$ directo al corazón $^{4,5,8}$. En nuestro hospital se realiza principalmente esternotomía media, ya que permite una excelente exposición a estructuras del mediastino anterior y grandes vasos intrapericárdicos, además, corresponde a un procedimiento sencillo y es el abordaje enseñado y practicado por la escuela de cirugía de nuestro hospital.

La toracotomía de urgencia en box ha sido considerada como una opción de resucitación frente a pacientes que ingresan a urgencias sin signos vitales, sin embargo, su supervivencia no supera el $21 \% \%^{4,5,10,26}$. Numerosas publicaciones se han dedicado a buscar predictores de supervivencia en pacientes reanimados con una toracotomía de urgencia en box, demostrándose una mayor resucitación y supervivencia en traumatismo penetrante versus contuso ${ }^{4,5,21}$. También se ha demostrado como predictores positivos los traumatismo por arma blanca versus por arma de fuego, teniendo este último hasta 11 veces mayor riesgo de mortalidad $^{4,5,26}$. Otro factor importante a considerar en este procedimiento son las secuelas neurológicas que se pueden presentar. Seamon y col, contactaron 37 pacientes sobrevivientes 11 años posterior a la toracotomía de urgencia en box, a los cuales se realizó una evaluación general, observándose en un $74 \%$ alteraciones sociales, cognitivas, funcionales o psicológicas posterior al traumatismo ${ }^{26}$. En nuestra serie se realizaron 2 toracotomías de urgencia en box no registrándose mortalidad ni déficit neurológico inmediato evidente. Nosotros, dadas las características de nuestro hospital, no recomendamos la realización de toracotomías de urgencia en box de rutina.

Se presentó una morbilidad similar a lo reportado en la literatura, donde se describen hemotórax postoperatorio, infecciones de tracto respiratorio, déficit neurológico permanente, entre otros ${ }^{19}$. En nuestro medio se presentó principalmente hemotórax y hemorragias postoperatorias con necesidad de reoperación en el 9,7\% de los casos. Morse y col, describieron la necesidad de transfusiones masivas en el $36 \%$ de los casos con una media de 16,5 UGR por paciente ${ }^{4}$. En nuestra serie se transfundieron el $33,6 \%$, con una media de 9,1 UGR por paciente transfundido.

La mayoría de los TPC (80-90\%) fallecen antes de ingresar a un servicio de urgencia, siendo una de las principales causas de mortalidad en el traumatismo torácico penetrante ${ }^{4,5,8-10}$. En nuestra serie la mortalidad operatoria fue algo menor a lo reportando tanto a nivel nacional (13-17\%) como internacional (16-42\%). Probablemente esta mortalidad se deba a una mejor atención pre-hospitalaria, rapidez de traslado, capacitación, experiencia y entrenamiento de los cirujanos, de urgencia, cardio y/o torácicos, que nos enfrentamos a este tipo de traumatismo ${ }^{27}$.

Distintas series han buscado factores predictores a morbimortalidad en pacientes operados por TPC. La evolución con shock y las lesiones por arma de fuego se han asociado a un peor pronóstico ${ }^{15}$. En nuestra serie el agente traumático no se asoció significativamente a mayor morbilidad ni mortalidad. La presencia de shock al ingreso o el cursar con un paro cardiorrespiratorio se identificaron como los principales factores asociados a mortalidad.

En conclusión, el traumatismo penetrante cardiaco, afecta principalmente a hombres jóvenes, secundario a una agresión por arma blanca. Se presenta clínicamente con taponamiento cardiaco en la mayoría de los casos, sin necesidad de algún método de imagen o procedimiento para su diagnóstico. Generalmente se produce lesión de las cavidades ventriculares, principalmente derecha, asociándose a una importante morbimortalidad. En nuestra serie identificamos variables asociadas a morbilidad, deterioro neurológico y mortalidad. Nuestros resultados quirúrgicos inmediatos son comparables con otras series. 


\section{Responsabilidades éticas}

Protección de personas y animales. Los autores declaran que para esta investigación no se han realizado experimentos en seres humanos ni en animales.
Confidencialidad de los datos. Los autores declaran que en este artículo no aparecen datos de pacientes.

Conflictos de interés: no hay.

\section{Referencias}

1. Asensio J, Ceballos J, Forno W, Torcal J, Gambaro E, Chahwan S, et al. Lesiones cardíacas penetrantes: una revisión desde sus orígenes históricos hasta las últimas fronteras en el nuevo milenio. Cir Esp. 2000;67:64-79.

2. Hernández R. Ludwig Rehn: las virtudes de un pionero. Cir Cardiov. 2008;15:2116.

3. Echevarría J, San Román A. Evaluación y tratamiento de los traumatismo cardíacos. Rev Esp Cardiol. 2000;53:727-35.

4. Morse B, Mina M, Carr J, Jhunjhunwala $\mathrm{R}$, Dente C, Zink J, et al. Pentranting cardiac injuries: a 36-year perspective at an urban, Level I trauma center. J Trauma Acute Care Surg. 2016;81:623-31.

5. Tavares B, Baltazar V, Rodríguez T, Pinheiro M, Petrucci O, Pereira G. Penetrating cardiac trauma: $20-\mathrm{y}$ experience from a university teaching hospital. JSR 2013;183:792-7.

6. Keel M, Trenz O. Pathophysiology of polytrauma. Injury Int J Care Injured 2005;36:691-709.

7. Mica L, Rufibach K, Keel M, Trentz O. The risk of early mortality of polytrauma patients associated to ISS, NISS, APACHE II values and prothrombin time. J Trauma Manag Outcome 2013;7:6. Published online 2013 May 24. doi: [10.1186/1752-2897-7-6].

8. De la Fuente H, Saa E, Zepeda N, Bucheister M, Villarroel L, Pefaur J. Herida penetrante cardiaca. Experiencia en 100 casos operados. Rev Chil Cir. 1993;45:229-34.

9. Rodríguez A, Furlanetti L, Faidiga G, Scarpelini S, Barbosa P, de Andrade W.
Penetrating Cardiac injuries: a 13-year retrospective evaluation from a Brazilian trauma center. Interact Cardiovasc Thorac Surg 2005;4:212-5.

10. Campbell N, Thomson S, Muckart D, Neumann C, Van Middelkoop I, Botha J. Review of 1198 cases of penetrating cardiac trauma. BJM 1997;84:1737-40.

11. Saldías R, González R, Núñez E, Alarcón E, Santander C, Seguel E, et al. Proyectiles cardíacos. Rev Chil Cir. 2003;55: 70-4.

12. Spodick D. Acute Cardiac Tamponade. $\mathrm{N}$ Engl J Med. 2003;349:684-90.

13. Moreno C, Moore E, Majure J, Hopeman A. Pericardial tamponade: A critical determinant for survival following penetrating cardiac wounds. J Trauma 1986;26: 821-5.

14. Morales CH, Salinas CM, Henao CA, Patiño PA, Muñoz CM. Thoracoscopic pericardial window and penetrating cardiac trauma. J Trauma 1997;42:273-5.

15. Asensio J, Murray J, Demetriades D, Berne J, Velmahos G, Gómez H, et al. Penetrating cardiac injuries: a prospective study of variables predicting outcomes. J Am Coll Surg. 1998;186:24-34.

16. Attar S, Suter C, Hankins J, Sequeira A, Mc Laughlin J. Penetrating Cardiac Injuries. Ann Thorac Surg. 1991;51: 711-6.

17. Saadia R, Levy R, Degiannis E, Velmahos G. Penetrating cardiac injuries: clinical classification and management strategy. BJS 1994;81:1572-5.

18. Kaplan AJ, Norcross ED, Crawford FA. Predictors of mortality in penetrating cardiac injury. Am Surg. 1993;59:338-41.

19. Rozycki GS, Feliciano DV, Schmidt JA, Cushman JG, Sisley AC, Ingram W, et al.
The role of surgeon-performed ultrasound in patients with possible cardiac wounds. Ann Surg. 1996;223:737-46.

20. Asencio J, Petrone P, Karida T, Kelly R, Roldan G, Park-art R, et al. Penetrating cardiac injuries. Complex injuries and difficult challenges. Ulus Trauma Derg. 2003;9:1-16.

21. O'Connor J, Ditillo M, Scalea T. Penetrating Cardiac Injury. JR Army Med Corps 2009;155:185-90.

22. Petre R, Chilcott M. Blunt Trauma to the Heart and Great Vassels. N Engl J Med. 1997;336:626-32.

23. Asensio J, Berne J, Demetriades D, Chan L, Murray J, Falabella A, et al. One hundred five penetrating cardiac injuries: A 2-year prospective evaluation. J Trauma 1998;44:1073-82.

24. González R, Zalaquett R, Chamorro G, Lema $\mathrm{G}$. Rotura de válvula tricúspide con insuficiencia masiva secundaria a traumatismo torácico cerrado. Caso clínico. Rev Med Chile 2008;136:1034-8.

25. González R, Santander C, Seguel E, Stockins A, Álvarez R, Cárdenas P, et al. Cierre espontáneo de fístula residual post cierre quirúrgico de fístula aortoventricular derecho traumática. Caso clínico. Rev Med Chile 2010;138:213-6.

26. Seamon M, Chovanes J, Fox N, Greem R, Manis G, Tsiotsias G, et al. The use of emergency department thoracotomy for traumatic cardiopulmonary arrest. Injury Int J Care Injured 2012;43:1355-61.

27. González R, Riquelme A, Fuentes A, Saldías R, Reyes R, Seguel E, et al. Traumatismo torácico: caracterización de hospitalizaciones durante tres décadas. Rev Med Chile 2018;146:149-58. 